\title{
Foldseek: fast and accurate protein structure search
}

\author{
Michel van Kempen, ${ }^{1,}$ Stephanie S. Kim, ${ }^{2}$, ${ }^{\boxplus}$ Charlotte Tumescheit, ${ }^{2}$

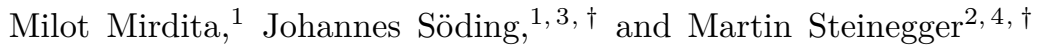

Highly accurate structure prediction methods are generating an avalanche of publicly available protein structures. Searching through these structures is becoming the main bottleneck in their analysis. Foldseek enables fast and sensitive comparisons of large structure sets. It reaches sensitivities similar to state-of-the-art structural aligners while being at least 20,000 times faster. Foldseek is free open-source software available at foldseek.com and as a webserver at search.foldseek.com.

Contact: soeding@mpinat.mpg.de, martin.steinegger@snu.ac.kr

1 The recent breakthrough in in-silico protein structure pre2 diction at near-experimental quality by AlphaFold2 [I] and ${ }_{3}$ then RoseTTAFold [ 2$]$ is revolutionizing structural biology 4 and bioinformatics. The European Bioinformatics Institute ${ }_{5}$ (EBI) in collaboration with AlphaFold2/DeepMind has al6 ready made $1,106,829$ protein structures publicly available 7 and plans to extend this library to hundreds of millions of 8 structures this year [3]. With these novel computational ap9 proaches, it will not be long before billions of high quality pro10 tein sequences become available [4]. The scale of this treasure 11 trove poses challenges to state-of-the-art analysis methods.

12 Currently, the most widely used approach to protein an13 notation and analysis is based on sequence similarity search $14[5-8]$. The goal is to find homologous sequences from which 15 one can infer properties of the query sequence, such as molec16 ular and cellular functions and structure. Despite the success 17 of sequence-based homology inference, many proteins cannot 18 be annotated because detecting distant evolutionary relation19 ships from sequences alone remains challenging [9].

20 Detecting similarity between protein structures by $3 \mathrm{D}$ su21 perposition offers higher sensitivity for identifying homolo22 gous proteins [III]. The imminent availability of high-quality 23 structure models for any protein of interest could allow us 24 to use structure comparison to improve homology-based in25 ference and structural, functional and evolutionary analyses. 26 However, despite decades of effort to improve speed and sen27 sitivity of structural aligners, current tools are much too slow 28 to cope with the expected scale of structure databases.

For example, searching with a single query structure 30 through a database with 100 million protein structures would 31 take the popular TMalign [II] tool around a month on one ${ }_{32} \mathrm{CPU}$ core, and an all-versus-all comparison would take around зз 10 millennia on a 1,000 core cluster. In comparison, sequence 34 searching is five orders of magnitude faster: An all-versus35 all comparison of $100 \mathrm{M}$ sequences would take MMseqs2 [6] at 36 high search sensitivity only around a week on the same cluster.

37 Structural alignment tools are slower for two reasons. First, 38 whereas sequence search tools employ fast and sensitive pre39 filter algorithms to gain several orders of magnitude in speed, 40 no comparable prefilters exist for structure searches. Sec-

\footnotetext{
* These two authors contributed equally

$\dagger$ Authors to whom correspondence should be addressed

${ }^{1}$ Quant. \& Comput. Biology, Max Planck Institute for Multidisciplinary Sciences, Göttingen, Germany. ${ }^{2}$ School of Biological Sciences, Seoul National University, Seoul, South Korea. ${ }^{3}$ Campus-Institute Data Science (CIDAS), Goldschmidtstrasse 1, 37077 Göttingen, Germany. ${ }^{4}$ Artificial Intelligence Institute, Seoul National University, Seoul, South Korea
}

${ }_{41}$ ond, structural similarity scores are non-local: changing the 42 alignment in one part affects the similarity in all other parts. ${ }_{43}$ For example in TMalign, two highly interdependent optimiza44 tions are performed: The pairing up of residues that are to be 45 aligned with each other, and the superposition of the 3D struc46 tures by minimizing some distance measure between aligned 47 residues. Most structural aligners, such as the popular TMa48 lign, DALI, and CE [1]-[3], solve the alignment optimization 49 problem by iterative or stochastic optimization.

To increase speed, a crucial idea is to describe the amino 51 acid backbone of proteins as sequences over a structural alpha52 bet and compare structures using sequence alignments [14]. In 53 this way, structural alphabets reduce structure comparisons to 54 much faster sequence alignments. Many ways to discretize the ${ }_{55}$ local amino acid backbone have been proposed [1.5]. Most, 56 such as CLE, 3D-BLAST, and Protein Blocks, discretize the 57 conformations of short stretches of usually 3 to $5 \mathrm{C}_{\alpha}$ atoms $58[16-18]$. 3D-BLAST and CLE trained a substitution matrix 59 for their structural alphabet and rely on an aligner like BLAST 60 [5] to perform the sequence searches.

For Foldseek, we developed a novel type of structural alpha${ }_{62}$ bet that does not describe the backbone but rather tertiary ${ }_{63}$ interactions. The 20 states of the $3 \mathrm{D}$-interactions (3Di) alpha${ }_{64}$ bet describe for each residue $i$ the geometric conformation with ${ }_{65}$ its spatially closest residue $j$. Compared to the various back66 bone structural alphabets, 3Di has three decisive advantages: ${ }_{67}$ First, the dependency of consecutive 3Di letters on each other 68 is much weaker than for backbone structural alphabets, where 69 for instance a helix state is followed by another helix state with 70 high probability. The dependency decreases information den71 sity and results in high-scoring false alignments. Second, the 72 frequencies of the 3Di states are more evenly distributed than 73 for backbone states, for which $60 \%$ describe generic secondary 74 structure states. This further increases information density in 75 3Di sequences and decreases false positives. Third, in back76 bone structural alphabets, less information is contained in the 77 highly conserved protein cores (consisting to a large part of 78 regular secondary structure elements) and more in the mostly 79 non-conserved coil/loop regions. In contrast, 3Di sequences 80 have the highest information density in the conserved core re81 gions and the lowest density in loop regions.

${ }_{82}$ Foldseek (Fig. Ta) (1) discretizes the query structures into 83 sequences over the 3Di alphabet and then searches through ${ }_{84}$ the $3 \mathrm{Di}$ sequences of the target structures using the double${ }_{85}$ diagonal $k$-mer-based prefilter and gapless alignment prefilter 86 modules from MMseqs2, our highly optimized and parallelized 87 open-source sequence search software [a]. (2) High scoring hits 


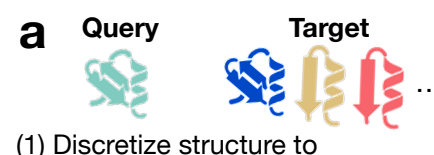

(1) Discretize structure to
sequence (b) and prefilter

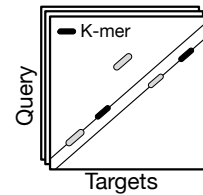

(2) Structural Alignment

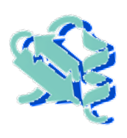

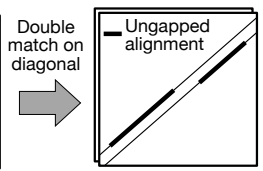

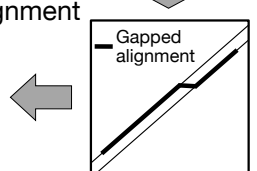

b

(1) Find neighboring residues using virtual center

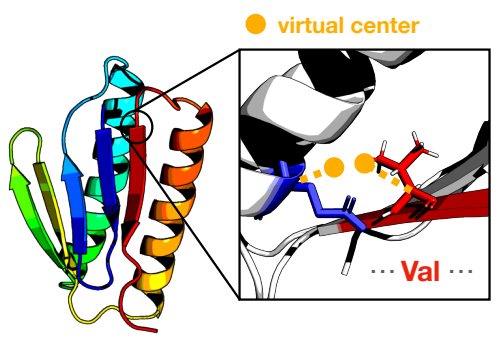

(2) Extract features

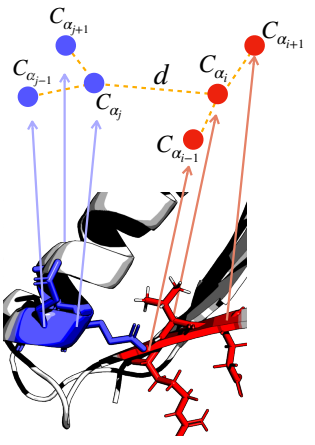

(3) Prediction stage: convert to 3Di sequence

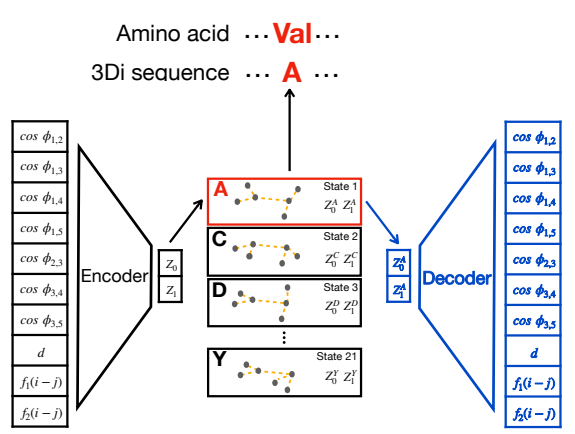

(3) Training stage: predict features by maximum likelihood

FIG. 1. Foldseek workflow. (a) Foldseek searches a set of query structures through a set of target structures. (1) Query and target structures are discretized into 3Di sequences (see b). To detect candidate structures, we apply the fast and sensitive $k$-mer and ungapped alignment prefilter from MMseqs2 on the 3Di sequences. (2) Followed by a local alignment using a vectorized Smith-Waterman algorithm combining both 3Di and amino acid substitution scores. Alternatively, a global alignment is computed with an accelerated TMalign version. (b) Learning the 3Di alphabet: (1) 3Di states describe tertiary interaction between a residue $i$ and its nearest neighbor $j$. Nearest neighbors have the closest virtual center distance (yellow). Virtual center (Supplementary Fig. 1) positions were optimized for maximum search sensitivity. (2) To describe the interaction geometry of residues $i$ and $j$, we extract seven angles, the euclidean $\mathrm{C}_{\alpha}$ distance, and two sequence distance features from the six $\mathrm{C}_{\alpha}$ coordinates of the two backbone fragments (blue, red). (3) These 10 features are used to define 20 3Di states by training a vector-quantized variational autoencoder [I.9] modified to learn states that are maximally evolutionary conserved. For structure searches, the encoder predicts the best-matching 3Di state for each residue.

88 are aligned locally (default) or aligned globally with TMa89 lign. The novel local alignment stage combines structural and 90 amino acid substitution scores for improved sensitivity with${ }_{91}$ out sacrificing speed. The construction of the 3Di alphabet is 92 summarized in Fig. $\mathbf{~} \mathbf{b}$ and Online Methods.

${ }_{93}$ To minimize high-scoring false positives and provide reli94 able E-values, for each match the score of the reversed query 95 sequence is subtracted from the original score. Furthermore, 96 a compositional bias correction is applied that lowers the sub97 stitution scores of 3Di states enriched within a local 40 residue 98 sequence window (see "Pairwise local structural alignments"). 99 E-values are calculated based on an extreme-value score dis100 tribution whose parameters are predicted by a neural network 101 from 3Di sequence composition and length (see "E-Values"?). 102 We measured the sensitivity and speed of Foldseek and six 103 structure alignment tools with single-domain structures (Fig. 104 [2/a-b) on the SCOPe40 dataset [20]. This dataset contains 10511,211 protein domains clustered at $40 \%$ sequence identity. ${ }_{106}$ We performed an all-versus-all search and compared the per${ }_{107}$ formance for finding members of the same SCOPe family, su108 perfamily, and fold (true positive matches, TPs) by measuring 109 the fraction of TPs out of all possible correct matches for the 110 query until the fifth false positive (FP). FPs are matches to a 111 different fold (see "SCOPe Benchmark' 112 measured by the area under the curve (AUC) of the cumula113 tive ROC curve up to the fifth FP.

Foldseek reaches sensitivities at family and superfamily 115 level below Dali, higher than the structural aligner CE, and 116 performs similarly to TMalign and TMalign-fast. Foldseek 117 is much more sensitive than structural alphabet-based search 118 tools 3D-BLAST and CLE-SW (Fig. 2/a-b). Even on the fold 119 level, where most TPs are between non-homologous superfam120 ilies, it is more sensitive than CE and similar to TMalign. Yet
${ }^{21}$ on this small, single-domain benchmark it is more than 3,000 122 times faster than TMalign, DALI, and CE (Fig. 2]b). On the 123 much larger AlphaFoldDB, where Foldseek approaches its full 124 speed, it is around 184,600 and 23,000 faster than DALI and ${ }_{125}$ TMalign, respectively (Fig. [2d). Its E-values are accurate, 126 which is critical for homology searching (Fig. [2/c)

${ }_{127}$ To assess the reliability and speed of Foldseek with full128 length protein chains, we performed an all-versus-all Foldseek 129 search on the AlphaFoldDB. For each query structure we com130 puted the TMalign score of Foldseek's second best match (the 131 best match is the self-match). We ignored matches for which 132 the average of the predicted Local Distance Difference Test ${ }_{133}$ (pLDDT [I] ) from query and target is below 80 or which are ${ }_{134}$ fragmented. All but 1,675 out of 133,813 second-best matches 135 with high alignment confidence (Foldseek score per aligned ${ }_{136}$ column $\left.\geq 1.0\right)$ had a good TM-score $(\geq 0.5)$, indicating that 137 the fold was correctly recognized (Supplementary Fig. 2). ${ }_{138}$ Manual inspection of outliers with high Foldseek score per col$139 \mathrm{umn}$ and low TMscores $(<0.5)$ revealed Foldseek matches with 140 multiple smaller, correctly aligned regions (Supplementary ${ }_{141}$ Table 1). Even though their average pLDDT is above 80, 142 the relative orientation of correctly folded segments is often 143 not correctly predicted by AlphaFold2. TM-align does not 144 identify these as homologs, as it looks for global structural 145 superpositions, thus overlooking significant local similarities.

146 We investigated the sensitivity for detecting very remote ho147 mologs by counting the number of cross-kingdom hits within ${ }_{148}$ AlphaFoldDB. In $12.2 \%$ of the 364,357 queries, Foldseek finds 149 cross-kingdom hits where MMseqs2 finds none. Overall, Fold150 seek finds 3.4 times as many cross-kingdom hits as MMseqs2 ${ }_{151}$ (see Supplementary Fig. 3).

${ }_{152}$ To facilitate access to Foldseek, we developed a user153 friendly webserver optimized to quickly return results for sin- 

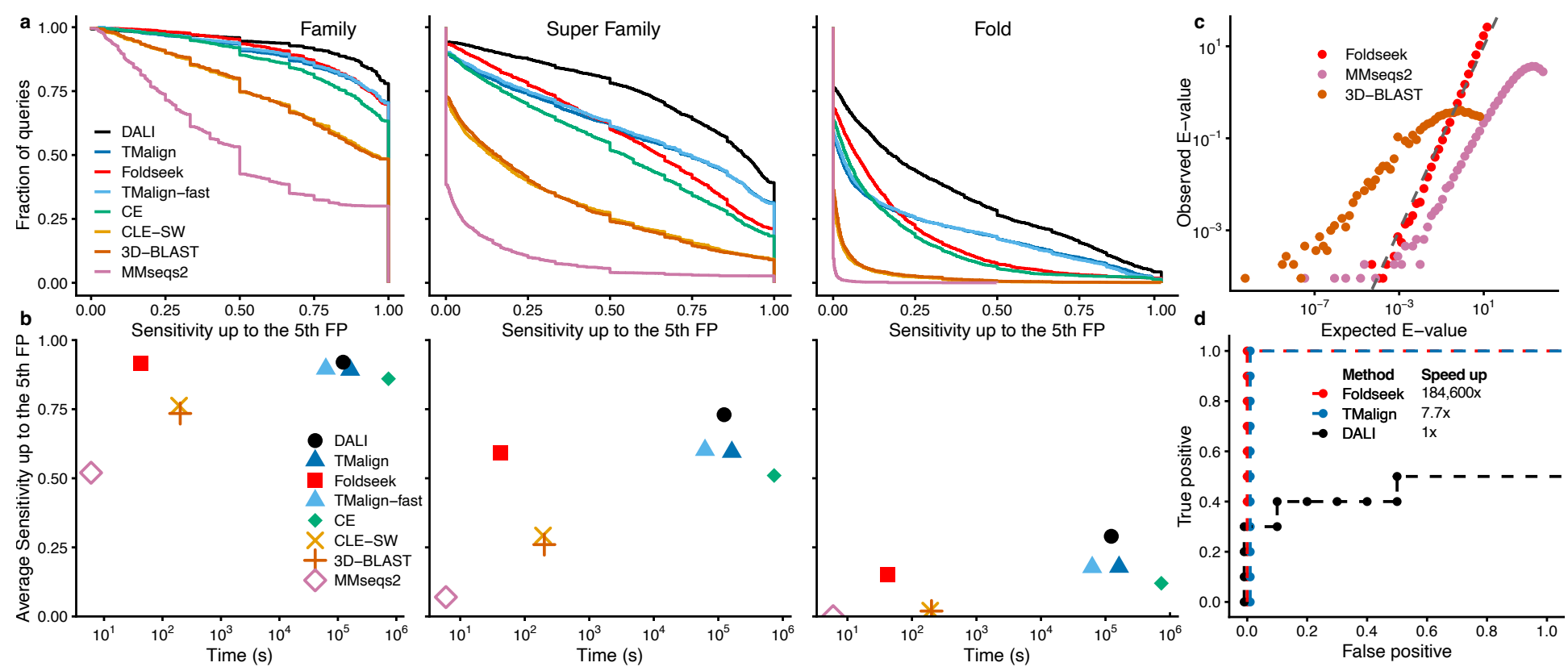

FIG. 2. Foldseek reaches similar sensitivities as structural aligners at thousands of times their speed (a) Cumulative distributions of sensitivity for homology detection. Sensitivity is the area under the ROC curve up to the fifth false positive, for all-versusall searches with the 11,211 single-domain structures of the SCOPe40 database). True positives are matches within the same family, superfamily or fold (see main text). (b) Sensitivity versus total runtime on an AMD EPYC 7702P 64-core CPU for the all-versus-all searches. (c) Accuracy of reported E-values: expected E-values versus observed E-values. (d) Top10 hits of search with RdRp (6M71_A) through the AlphaFold/Proteome with Foldseek, TMalign and DALI.

154 gle queries. It performs searches through three structure 185 with structures. The main limitation in our view, the four 155 databases, AlphaFoldDB/Proteome, AlphaFoldDB/Swiss- 186 orders of magnitude slower speed of structure comparisons, is 156 Prot, and the PDB100, using one of three alignment methods: 188 removed by Foldseek. ${ }_{157}$ standard Foldseek (default), Foldseek without amino acid scor158 ing, and TMalign. The server takes PDB files as input and re159 turns a list of matched structures, query-target sequence align160 ments, similarity scores, and significance (E-value/TMscore). ${ }_{161}$ We compared the Foldseek webserver with TMalign and 162 DALI by searching with the SARS-CoV-2 RNA-dependent ${ }_{193}$ ${ }_{163}$ RNA polymerase (RdRp, PDB: 6M71_A [ZI] ; 942 residues) 194 164 through the AlphaFoldDB (Proteome + Swiss-Prot) contain- ${ }^{195}$ 165 ing 804,872 protein structures. The searches took TMalign ${ }_{166} 33 \mathrm{~h}$ and DALI 10 days to complete on a single core. Foldseek ${ }_{167}$ took 5 seconds, which is about 23,000, 180,600 times faster 168 than TMalign and DALI respectively. We compared the top 16910 hits of the AlphaFoldDB/Proteome database (Fig. 2]d). 170 Foldseek as well as TMalign contain only reverse transcrip171 tase (RT) domains, which are structurally similar to RdRps. ${ }_{172}$ DALI finds three RdRp and two RT hits, and five FPs hits to 173 kinases (Supplementary Table 2). Foldseek finds significant 174 hits with E-values between $10^{-7}$ to $10^{-6}$, while TMalign re175 ports low TM-scores between 0.419 and 0.42 . This illustrates 176 a key difference between structural aligners, which depend on 177 finding a global 3D superposition, and Foldseek's local align178 ment. Foldseek is independent of the relative orientation of 179 domains and therefore excels at detecting homologous multi180 domain structures.

${ }_{181}$ The availability of high-quality protein structures for nearly 182 every structured protein is going to be transformative for ${ }_{183}$ structural biology and bioinformatics. What could until re- ${ }_{216}$

184 cently only be done by analyzing sequences can now be done 217 [21] Gao, Y. et al. Science 368, 779-782 (2020).

\section{REFERENCES}

189 [1] Jumper, J. et al. Nature 596, 583-589 (2021).

[2] Baek, M. et al. Science 373, 871-876 (2021).

[3] Varadi, M. et al. Nucleic Acids Res 50, D439-D444 (2022).

[4] Burley, S. K. et al. Nucleic Acids Res 49, D437-D451 (2021).

[5] Altschul, S. F. et al. J Mol Biol 215, 403-410 (1990).

[6] Steinegger, M. \& Söding, J. Nat Biotechnol 35, 1026-1028 (2017).

[7] Steinegger, M. et al. BMC Bioinform 20, 473 (2019).

[8] Buchfink, B. et al. Nat Methods 18, 366-368 (2021).

[9] Rost, B. Protein Eng Des Sel 12, 85-94 (1999).

99 [10] Illergård, K. et al. Proteins 77, 499-508 (2009).

200 [11] Zhang, Y. \& Skolnick, J. Nucleic Acids Res 33, 2302-2309 (2005).

02 [12] Holm, L. Methods Mol Biol 2112, 29-42 (2020).

203 [13] Shindyalov, I. N. \& Bourne, P. E. Protein Eng Des Sel 11, 739-747 (1998)

[14] Guyon, F. et al. Nucleic Acids Res 32, W545-W548 (2004).

[15] Ma, J. \& Wang, S. Adv Protein Chem Struct Biol 94, 121-175 (2014).

208 [16] Wang, S. \& Zheng, W.-M. J Bioinform Comput Biol 6, 347366 (2008).

[17] Yang, J.-M. \& Tung, C.-H. Nucleic Acids Res 34, 3646-3659 (2006).

2 [18] de Brevern, A. G. et al. Proteins 41, 271-287 (2000).

213 [19] Van den Oord, A. et al. Adv Neur Inf Proc Syst (NIPS) 30 (2017).

5 [20] Chandonia, J.-M. et al. Nucleic Acids Res 47, D475-D481 (2019). 
${ }_{219}$ We thank Nicola Bordin, Ian Sillitoe and Christine Orengo 220 for reporting issues and providing valuable feedback, and 221 Yang Zhang and Marcin Wojdyr for making TMalign and the 222 Gemmi library freely accessible.

${ }_{223}$ M.S. acknowledges support from the National Research ${ }_{224}$ Foundation of Korea grants [2019R1A6A1A10073437, 225 2020M3A9G7103933, 2021R1C1C102065, 2021M3A9226 I4021220]; and the Creative-Pioneering Researchers Program 227 through Seoul National University. S.K. acknowledges 228 support by the National Research Foundation of Korea 229 (NRF) grant No. 2019R1A6A1A10073437. M.M. and J.S. 230 acknowledge support by the German ministry for education 231 and research (BMBF) via grant horizontal4meta.

${ }_{232}$ This work used the Scientific Compute Cluster at GWDG, 233 the joint data center of Max Planck Society for the Advance234 ment of Science (MPG) and University of Göttingen.

\section{Author contributions}

${ }_{236}$ M.K., S.K., J.S. \& M.S. designed research. M.K., S.K., ${ }_{237}$ C.T., \& M.S. developed code and performed analyses. M.K. 238 and J.S. developed the 3Di library. M.M. developed the 239 webserver. M.K., S.K., C.T., M.M., J.S. \& M.S. wrote the 240 manuscript.

\section{Competing financial interests}

${ }_{242}$ The authors declare no competing financial interests. 


\section{METHODS}

${ }_{244}$ Overview Foldseek enables fast and sensitive comparison of 245 large structure sets. It encodes structures as sequences over 246 the 20-state 3Di alphabet and thereby reduces structural align247 ments to 3Di sequence alignments. The 3Di alphabet devel248 oped for Foldseek describes tertiary residue-residue interac249 tions instead of backbone conformations and proved critical 250 for reaching high sensitivities. Foldseek's prefilter finds two ${ }_{251}$ similar, spaced 3Di $k$-mer matches in the same diagonal of 252 the dynamic programming matrix. By not restricting itself to 253 exact matches, the prefilter achieves high sensitivity while re254 ducing the number of sequences for which full alignments are 255 computed by several orders of magnitude. Further speed-ups 256 are achieved by multi-threading and utilizing single instruction ${ }_{257}$ multiple data (SIMD) vector units. Owing to the SIMDe li${ }_{258}$ brary (github.com/simd-everywhere/simde), Foldseek runs on 259 a wide range of CPU architectures (x86_64, arm64, ppc64le) 260 and operating systems (Linux, macOS). The core modules of ${ }_{261}$ Foldseek, which build on the MMseqs2 framework [ [22], are described in the following paragraphs.

${ }_{263}$ Create database The createdb module converts a set of ${ }_{264}$ Protein Data Bank (PDB; [23]) or macromolecular Crystal${ }_{265}$ lographic Information File (mmCIF) formatted files into an 266 internal Foldseek database format using the gemmi package 267 (project-gemmi.github.io). The format is compatible with the ${ }_{268}$ MMseqs2 database format, which is optimized for parallel ac269 cess. We store each chain as a separate entry in the database. 270 The module follows the MMseqs 2 createdb module logic, 271 however, in addition to the amino acid sequence it computes 272 the $3 \mathrm{Di}$ sequence from the $3 \mathrm{D}$ atom coordinates of the $\mathrm{C}_{\alpha}$ and 巴a $\mathrm{C}_{\beta}, \mathrm{C}_{\text {backbone }}$ and $\mathrm{N}$ coordinates (see "Learning 3Di states us274 ing $\mathrm{VQ}$ - $\left.\mathrm{VAE} \mathrm{F}^{\prime}\right)$. The $3 \mathrm{Di}$ and amino acid sequence, and the $\mathrm{C}_{\alpha}$ floating-point coordinates are stored in the database.

${ }_{276}$ Prefilter The prefilter module generates similar k-mers 277 and detects double, consecutive, similar k-mer matches that 278 occur on the same diagonal. In contrast to the MMsegs2 279 prefilter, the Foldseek prefilter utilizes the 3Di information 280 instead of the amino acid sequence information to generate

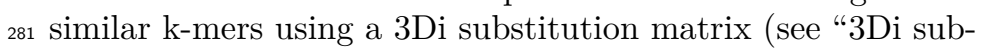
282 stitution score matrix"). This criterion suppresses hits to 283 non-homologous structures effectively, as they are less likely 284 to have consecutive k-mer matches on the same diagonal by 285 chance. To counteract the effect of regions with 3Di compo286 sitions that differ from the database average, a compositional 287 bias correction is applied in a way analogous to MMseqs2 [24]. ${ }_{288}$ For each hit we perform an ungapped alignment over the di289 agonals with double, consecutive, similar k-mer matches and 290 sort those by the maximum ungapped diagonal score. Align291 ments with a score of at least 15 bits are passed onto the next stage.

${ }_{293}$ Pairwise local structural alignments After the prefilter 294 has removed the vast majority of non-homologous sequences, 295 pairwise alignments are performed on the remaining sequences 296 in the structurealign module. Sequences are aligned us297 ing a SIMD accelerated Smith-Waterman algorithm [25, 26] ${ }_{298}$ We extended this implementation to support amino acid and
299 3Di scoring, compositional bias correction, and 256-bit-wide 300 vectorization. The score linearly combines amino acid and ${ }_{301} 3 \mathrm{Di}$ substitution scores with weights 1.4 and 2.1 , respectively. 302 A compositional bias correction is applied to the amino acid 303 and 3Di scores. To further suppress high-scoring false positive 304 matches, for each match we align the reversed query sequence 305 against the target and subtract the reverse score from the forward score.

E-Values To estimate E-values for each match, we trained a ${ }_{0}$ neural network to predict the mean $\mu$ and scale parameter $\lambda$ of 309 the extreme value distribution for each query. We built a mod310 ule in Foldseek called computemulambda, which takes a query 311 and database structures as input and aligns the query against a 312 randomly shuffled version of the database sequences. For each 313 query sequence the module produces $N$ random alignments 314 and fits to their scores an extreme-value (Gumbel) distribu315 tion. The maximum likelihood fitting is done using the Gum316 bel fitting function taken from HMMER3 ( hmmcalibrate) 317 [27]. To train the network, we predicted $\mu$ and $\lambda$ for 100,000 318 sequences sampled from the AlphaFoldDB. We trained the 319 network to predict $\mu$ and $\lambda$ from the mono-residue composi320 tion of the query and its length. The network has 22 input 321 nodes, 2 fully-connected layers with 32 nodes each (ReLU ac322 tivation) and two linear output nodes. The optimizer ADAM ${ }^{323}$ with learning rate 0.001 was used for training. When testing 324 the resulting E-values on searches with scrambled sequences, 325 the $\log$ of the expected number of false positives turned out 326 to have an accurately linear dependence on the log of the E${ }_{327}$ values, albeit with a slope of 0.32 instead of 1 . We therefore 328 correct the E-values from the neural network by taking them 329 to the power of 0.32 . We compared how well the observed 330 number of FPs at a given E-value agreed with the E-values 331 reported by Foldseek, MMseqs2, and 3D-Blast, (Fig. Z $\mathbf{c}$ for ${ }_{332}$ SCOPe and Supplementary Fig. 4 for AlphaFoldDB). We 333 considered a hit as FP if it was in a different fold and had a 334 TM-score lower than 0.3. Furthermore, we ignored all cross335 fold hits within the four- to eight-bladed $\beta$-propeller super336 families (SCOPe b.66-b.70) and within the Rossman-like folds ${ }_{337}$ (c.2-c.5, c.27, c.28, c.30, and c.31) because of the extensive cross-fold homologies within these groups [28].

${ }_{339}$ Pairwise global structural alignments using TM-align 340 We also offer the option to use TM-align for pairwise align${ }_{341}$ ments. We implemented TM-align based on the $\mathrm{C}_{\alpha}$ atom co342 ordinates and made adjustments to improve the (1) speed and 343 (2) memory usage. (1) TM-align performs multiple floating344 point based Needleman-Wunsch (NW) alignment steps, while 345 applying different scoring functions (e.g., score secondary 346 structure, Euclidean distance of superposed structures or frag347 ments, etc.) TM-align's NW code did not take advantage of ${ }_{348}$ SIMD instructions, therefore, we replaced it by parasail's [2.9] ${ }_{349}$ SIMD-based NW implementation and extended it to support 350 the different scoring functions. We also replaced the TM-score 351 computation using fast_protein_cluster's SIMD based imple352 mentation [30]. Our NW implementation does not compute 353 exactly the same alignment since we apply affine gap costs 354 while TM-align does not. (2) TMalign requires 17 bytes $\times$ 355 query length $\times$ target length of memory, we reduce the con356 stant overhead from 17 to 4 bytes. If Foldseek is used in TM- 
357 align mode (parameter --alignment-type 1), we replace the 358 reported E-value column with TM-scores normalized by the 359 query length. The results are ordered in descending order by ${ }_{360}$ TM-score.

${ }_{361}$ Descriptors for 3Di structural alphabet The 3Di alpha362 bet describes the tertiary contacts between residues and their ${ }_{363}$ nearest neighbors in 3D space. For each residue $i$ the con364 formation of the local backbone around $i$ together with the 365 local backbone around its nearest neighbor $j$ is approximated 366 by 20 discrete states. We chose the alphabet size $A=20$ as 367 a trade-off between encoding as much information as possible 368 (large $A$ ) and limiting the number of similar 3Di $k$-mers that 369 we need to generate in the $k$-mer based prefilter. This number 370 scales with $A^{k}$, giving us an alphabet size similar to the size 371 of the amino acid alphabet. The discrete single-letter states 372 are formed from neighborhood descriptors containing ten fea${ }_{373}$ tures encoding the conformation of backbones around residues ${ }_{374} i$ and $j$ represented by the $\mathrm{C}_{\alpha}$ atoms $\left(\mathrm{C}_{\alpha, i-1}, \mathrm{C}_{\alpha, i}, \mathrm{C}_{\alpha, i+1}\right)$ and 375 $\left(\mathrm{C}_{\alpha, j-1}, \mathrm{C}_{\alpha, j}, \mathrm{C}_{\alpha, j+1}\right)$. The descriptors use the five unit vec376 tors along the following directions,

$$
\begin{aligned}
& u_{1}: \mathrm{C}_{\alpha, i-1} \rightarrow \mathrm{C}_{\alpha, i} \quad u_{4}: \mathrm{C}_{\alpha, j} \rightarrow \mathrm{C}_{\alpha, j+1} \\
& u_{2}: \mathrm{C}_{\alpha, i} \rightarrow \mathrm{C}_{\alpha, i+1} \quad u_{5}: \mathrm{C}_{\alpha, i} \rightarrow \mathrm{C}_{\alpha, j} \\
& u_{3}: \mathrm{C}_{\alpha, j-1} \rightarrow \mathrm{C}_{\alpha, j} .
\end{aligned}
$$

${ }_{377}$ We define the angle between $u_{k}$ and $u_{l}$ as $\phi_{k l}$, so $\cos \phi_{k l}=u_{k}^{T} u_{l}$. ${ }_{378}$ The seven features $\cos \phi_{12}, \cos \phi_{34}, \cos \phi_{15}, \cos \phi_{35}, \cos \phi_{14}$, ${ }_{379} \cos \phi_{23}, \cos \phi_{13}$, and the distance $\left|\mathrm{C}_{\alpha, i}-\mathrm{C}_{\alpha, j}\right|$ describe the 380 conformation between the backbone fragments. In addition, 381 we encode the sequence distance with the two features $\operatorname{sign}(i-j) \min (|i-j|, 4)$ and $\operatorname{sign}(i-j) \log (|i-j|+1)$.

${ }_{383}^{382}$ Learning the 3Di states using a VQ-VAE The ten384 dimensional descriptors were discretized into an alphabet 385 of 20 states using a variational autoencoder with vector386 quantized latent variables (VQ-VAE) [3]. In contrast to ${ }_{387}$ the standard VQ-VAE, we trained the VQ-VAE not as a 388 simple generative model but rather to learn states that are ${ }_{389}$ maximally conserved in evolution. To that end, we trained 390 it with pairs of descriptors $\mathbf{x}_{n}, \mathbf{y}_{n} \in \mathbb{R}^{10}$ from structurally 391 aligned residues, to predict the distribution of $\mathbf{y}_{n}$ from $\mathbf{x}_{n}$. 392 The VQ-VAE consists of an encoder and decoder network 393 with the discrete latent 3Di state as a bottleneck in-between. 394 The encoder network embeds the 10-dimensional descriptor ${ }_{395} \mathbf{X}_{n}$ into a two-dimensional continuous latent space, where the 396 embedding is then discretized by the nearest centroid, each 397 centroid representing a 3Di state. Given the centroid, the 398 decoder predicts the probability distribution of the descriptor ${ }_{399} \mathbf{y}_{n}$ of the aligned residue. After training, only encoder and 400 centroids are used to discretize descriptors. Encoder and 401 decoder networks are both fully connected with two hidden 402 layers of dimension 10, a batch normalization after each 403 hidden layer and ReLU as activation functions. The encoder, 404 centroids, and decoder have 242, 40, and 352 parameters, ${ }_{405}$ respectively. The output layer of the decoder consists of 20 406 units predicting $\mu$ and $\sigma^{2}$ of the descriptors $x$ of the aligned ${ }_{407}$ residue, such that the decoder predicts $\mathcal{N}\left(x \mid \mu, I \sigma^{2}\right)$ (with ${ }_{408}$ diagonal covariance). We trained the VQ-VAE on the loss 409 function defined in Equation (3) in [3] (with commitment
${ }_{410}$ loss $\left.=0.25\right)$ using the deep-learning framework PyTorch 411 (version 1.9.0), the ADAM optimizer, with a batch size 412 of 512 , and a learning rate of $10^{-3}$ over 4 epochs. Using ${ }_{413}$ Kerasify, we integrated the encoder network into Foldseek. ${ }_{414}$ The domains from the SCOPe database were split 80\%/20\% 415 by fold into training and validation sets. For the training, we 416 structurally aligned the structures with TMalign, removed 417 all alignments with a TM-score below 0.6 , and removed all ${ }_{418}$ aligned residue pairs with a distance between their $\mathrm{C}_{\alpha}$ atoms 419 of more than $5 \AA$. We trained the VQ-VAE with 100 different 420 initial parameters and chose the model that was performing 421 best in the benchmark on the validation dataset (the highest 422 sum of ratios between 3Di AUC and TMalign AUC for family, superfamily and fold level).

${ }_{424} 3$ Di substitution score matrix We trained a BLOSUM425 like substitution matrix for 3Di sequences from pairs of 426 structurally aligned residues used for the "VAE- VQ training"]. ${ }_{427}$ First, we determined the 3Di states of all residues. Next, the 428 substitution frequencies between 3Di states were calculated 429 by counting how often two 3Di states were structurally 430 aligned. (Note that the substitution frequencies from state $\mathrm{A}$ 431 to B and the opposite direction are equal.) Finally, the score ${ }_{432} \mathrm{~S}(x, y)=2 \log _{2} \frac{p(x, y)}{p(x) p(y)}$ for substituting state $\mathrm{x}$ through state ${ }_{433} \mathrm{y}$ is the log-ratio between the substitution frequency $p(x, y)$ ${ }_{434}$ and the probability that the two states occur independently, scaled by the factor 2 .

${ }_{436}$ Optimize nearest-neighbor selection To select nearest${ }_{437}$ neighbor residues that maximize the performance of the ${ }_{438}$ resulting 3Di alphabet in finding and aligning homologous 439 structures, we introduced the virtual center $V$ of a residue. ${ }_{440}$ The virtual center position is defined by the angle $\theta(V$ $\left.{ }_{441} \mathrm{C}_{\alpha}-\mathrm{C}_{\beta}\right)$, the dihedral angle $\tau\left(V-\mathrm{C}_{\alpha}-\mathrm{C}_{\beta}-\mathrm{N}\right)$, and the length ${ }_{442} l\left(\left|V-\mathrm{C}_{\alpha}\right|\right)$. For each residue $i$ we selected the residue $j$ 443 with the smallest distance between their virtual centers. The 444 virtual center was optimized on the training and validation 445 structure sets used for the VQ-VAE training by creating 446 alphabets for positions with $\theta \in[0,2 \pi], \tau \in[-\pi, \pi]$ in $45^{\circ}$ ${ }_{447}$ steps, and $l \in\{1.53 \AA k: k \in\{1,1.5,2,2.5,3\}\} \quad(1.53 \AA$ is the 448 distance between $\mathrm{C}_{\alpha}$ and $\mathrm{C}_{\beta}$ ). The virtual center defined by ${ }_{449} \theta=270^{\circ}, \tau=0^{\circ}$ and $l=2$ performed best in the benchmark. ${ }_{450}$ For glycines, the $\mathrm{C}_{\beta}$ positions were approximated by forming ${ }_{451}$ a tetrahedral from $\mathrm{C}_{\alpha}$. This virtual center preferably selects 452 long-range, tertiary interactions and only falls back to 453 selecting interactions to $i+1$ or $i-1$ when no other residues 454 are nearby. In that case, the interaction captures only the 455 backbone conformation.

${ }_{456} \mathrm{SCOPe}$ Benchmark We downloaded SCOPe 2.07 [32] ${ }_{457}$ structures, clustered at $40 \%$ sequence identity, containing 45811,211 domains, for the generation of 3Di states and for the ${ }_{459}$ performance evaluation of Foldseek. The SCOPe benchmark 460 set consists of single domains with an average length of 174 ${ }_{461}$ residues. In our benchmark, we compare the domains all462 versus-all. Per domain, we measured the fraction of detected ${ }_{463}$ TPs up to the 5th false positive. For family-, superfamily464 and fold-level recognition, TPs were defined as same family, 465 same superfamily and not same family, and same fold and 466 not same superfamily, respectively. Hits from different folds are FPs. 
${ }_{467}$ AlphaFold database used for all-versus-all search We ${ }_{468}$ downloaded the AlphaFoldDB (reference) version 1 containing ${ }_{469} 365,198$ protein models and searched it all-versus-all using ${ }_{470}$ Foldseek -s 9.5 --max-seqs 2000. For our second best 471 hit analysis we consider only models with: (1) an average ${ }_{472} \mathrm{C}_{\alpha}$ 's pLDDT greater than or equal to 80 , and (2) models of 473 non-fragmented domains. We also computed the structural similarity for each pair using TMalign (default options).

${ }_{475}^{474}$ Performance evaluation: Sensitivity In order to evaluate 476 the sensitivity of the structural alignment tools, we used a ${ }_{477}$ cumulative ROC curve analysis. After sorting the alignment ${ }_{478}$ result of each query, we calculated the fraction of TPs in the ${ }_{479}$ list up to the 5 th false positives. We quantitatively measured ${ }_{480}$ the sensitivity by comparing the area under the curve (AUC) for family-, superfamily-, and fold-level classifications.

${ }_{482}^{481}$ Performance evaluation: Runtime Using the SCOPe ${ }_{483}$ benchmark dataset, the runtime of the pairwise structural ${ }_{484}$ alignment was evaluated for all methods. Depending on the ${ }_{485}$ processing time of each tool, the runtimes of the structural ${ }_{486}$ alignment tools TM-align, DALI, and CE were estimated on ${ }_{487} 10 \%$ of the benchmark set $(1,121$ proteins randomly selected ${ }_{488}$ from the SCOPe domains). Tools with multi-threading sup${ }_{489}$ port (MMseqs2 and Foldseek) were executed with 64 threads, 490 tools without were parallelized by breaking the query set into 64 equally sized chunks and executing them in parallel.

${ }_{492}$ Tools and options for benchmark comparison Following ${ }_{493}$ are command lines used in the SCOPe benchmark.

${ }_{494}$ Foldseek We used Foldseek commit 4de45 during this 495 analysis. Foldseek was run with the following parameters: --threads 64 -s 9.5 -e 10 --max-seqs 2000

${ }_{497}^{496}$ MMseqs2 We used the default MMseqs2 (release 13-45111) 498 search algorithm to obtain the sequence-based align${ }_{499}$ ment result. MMseqs2 sorts the results by e-value and 500 score. We searched with: --threads 64 -s 7.5 -e 10000 ${ }_{501}$--max-seqs 2000

${ }_{502}^{501}$ CLE-Smith-Waterman We used PDB Tool v4.80 ${ }_{503}$ (github.com/realbigws/PDB_Tool) to convert the bench${ }_{504}$ mark structure set to CLE sequences. After the conversion, ${ }_{505}$ we used SSW [26] (commit ad452e) to align CLE sequences 506 all-versus-all. We sorted the results by alignment score. The ${ }_{507}$ following parameters were used to run SSW: (1) protein 508 alignment mode (-p), (2) gap open penalty of 100 (-o 100), ${ }_{509}$ (3) gap extend penalty of 10 (-e 10 ), (4) CLE's optimized 510 substitution matrix (-a cle.shen.mat), (5) returning align${ }_{511}$ ment $(-c)$. The gap open and extend values were inferred ${ }_{512}$ from DeepAlign [33]. The results are sorted by score in 513 descending order.

ssw_test -p -o 100 -e 10 -a cle.shen.mat -c

${ }_{515}$ 3D-BLAST We used 3D-BLAST (beta102) with BLAST+ ${ }_{516}(2.2 .26)$ and SSW [26] (version ad452e). We first converted 517 the PDB structures to a 3D-BLAST database using 3d-blast 518 -sq_write and 3d-blast -sq_append. We searched the 519 structural sequences against the database using blastp ${ }_{520}$ with the following parameters: (1) we used 3D-BLAST's ${ }_{521}$ optimized substitution matrix (-M 3DBLAST), (2) number of 522 hits and alignments shown of 12,000 (-v 12000 -b 12000), ${ }_{523}$ (3) E-value threshold of 1,000 (-e 1000) (4) disabling query
524 sequence filter (-F F) (5) gap open of 8 (-G 8), and (6) gap ${ }_{525}$ extend of 2 (-E 2). 3D-BLAST's results are sorted by E-value 526 in ascending order:

527 blastall -p blastp -M 3DBLAST -v 12000 -b 12000 -e $5281000-F$ F $-G 8$-E 2

529 For Smith-Waterman we used (1) gap open of 8 (2) gap 530 extend of 2 and (3) returning alignments (-c) (4) using the ${ }_{531}$ 3D-BLAST's optimized substitution matrix (-a 3DBLAST), 532 (5) protein alignment mode (-p): ssw_test -o 8 -e 2 -c 533 -a 3DBLAST - p. Presented in Figure 2 are the Smith${ }_{534}$ Waterman results, since BLAST performed worse with an 535 average AUC of $0.573,0.127,0.009$ for family-, superfamilyand fold-classification, respectively.

${ }_{537}$ TMalign We downloaded and compiled the TMalign.cpp 538 source code (version 2019/08/22) from the Zhang group 539 website. We ran the benchmark using default parameters and ${ }_{540}$ - $f$ ast for the fast version. We used the TM score normalized 541 by the 1st chain (query) in all our analyses. Default: TMalign ${ }_{542}$ query.pdb target.pdb

Fast: TMalign query.pdb target.pdb -fast

${ }_{544}^{543}$ DALI We installed the standalone DaliLite.v5. For the ${ }_{545}$ SCOPe benchmark set, input files were formatted in DAT ${ }_{546}$ files with DALI's import.pl. The conversion to DAT format ${ }_{547}$ produced 11,137 valid structures out of the 11,211 initial 548 structures for the SCOPe benchmark. After formatting the 549 input files, we calculated the protein alignment with DALI's 550 structural alignment algorithm. The results were sorted by ${ }_{551}$ DALI's Z-score:

552 import.pl -pdbfile query.pdb -pdbid PDBid -dat DAT

${ }_{553}$ dali.pl -cd1 queryDATid -db targetDB.list -TITLE

554 systematic -dat1 DAT -dat2 DAT -outfmt "summary" -clean

${ }_{556} \mathbf{C E}$ We used BioJava's [34] (version 5.4.0) implementation 557 of the combinatorial extension (CE) alignment algorithm. ${ }_{558}$ We modified one of the modules of BioJava under shape 559 configuration to calculate the $\mathrm{CE}$ value. Our modified ${ }_{560}$ CEalign.jar file requires a list of query files, path to the ${ }_{561}$ target PDB files, and an output path as input parameters. ${ }_{562}$ This Java module runs an all-versus-all CE calculation. The ${ }_{563}$ Jar file of our implementation of CE calculation is provided.

${ }_{564}$ java -jar CEalign.jar querylist.txt

\section{TargetPDBDirectory OutputDirectory}

566 Hardware specifications for benchmarks The runtime 567 benchmarks were executed on a machine with an AMD EPYC 7702P 64-core CPU and 1024 GB RAM memory.

${ }_{569}$ Webserver The Foldseek webserver is a continuation of the ${ }_{570}$ MMseqs2 webserver [35]. To allow for searches in seconds 571 we implemented MMseqs2's pre-computed database indexing 572 capabilities in Foldseek. Using these, the search databases can 573 be held fully in system memory by the operating system and 574 instantly accessed by each Foldseek process, thus avoiding ex${ }_{575}$ pensive accesses to slow disk drives. A similar mechanism was 576 used to store and read the associated taxonomic information. ${ }_{577}$ The AlphaFoldDB/Proteome (v1), AlphaFoldDB/Swiss-Prot 578 (v2), and PDB100 require 3.9GB, 3.6GB, and 2.2GB RAM, 579 respectively. The databases are kept in memory using vmtouch (github.com/hoytech/vmtouch). 
${ }_{581}$ Code availability Foldseek is GPLv3-licensed free open 582 source software. The source code and binaries for Foldseek can 583 be downloaded at github.com/steineggerlab/foldseek. 584 The webserver code is available at github.com/soedinglab/ 585 mmseqs2-app. The analysis scripts are available at: github.com/steineggerlab/foldseek-analysis.

${ }_{587}^{58}$ Data availability Benchmark data and Foldseek databases ${ }_{588}$ are available at: wwwuser.gwdg.de/ compbiol/foldseek.

589

\section{REFERENCES}

590 [22] Steinegger, M. \& Söding, J. Nat Biotechnol 35, 1026-1028 $591 \quad$ (2017).

592 [23] Burley, S. K. et al. Nucleic Acids Res 47, D520-D528 (2019).

593 [24] Hauser, M. et al. Bioinformatics 32, 1323-1330 (2016).

594 [25] Farrar, M. Bioinformatics 23, 156-161 (2007).

595 [26] Zhao, M. et al. PLOS One 8, e82138 (2013).

596 [27] Eddy, S. R. PLOS Comput Biol 7, e1002195 (2011).

597 [28] Söding, J. \& Remmert, M. Curr Opin Struct Biol 21, 404-411 $598 \quad(2011)$.

599 [29] Daily, J. BMC Bioinform 17, 81 (2016).

600 [30] Hung, L.-H. \& Samudrala, R. Bioinformatics 30, 1774-1776 $601 \quad(2014)$.

602 [31] Van den Oord, A. et al. Adv Neur Inf Proc Syst (NIPS) 30 $603 \quad$ (2017).

604 [32] Chandonia, J.-M. et al. Nucleic Acids Res 47, D475-D481 605 (2019).

606 [33] Jiménez-Moreno, A. et al. J Struct Biol 213, 107712 (2021).

607 [34] Lafita, A. et al. PLOS Comput Biol 15, e1006791 (2019).

608 [35] Mirdita, M. et al. Bioinformatics 35, 2856-2858 (2019). 